\title{
Ludovico Carracci a Roma
}

\begin{abstract}
This essay focuses on Ludovico Carracci (1555-1619) and his reputation in Rome in his lifetime and afterwards. Well-known, but often overlooked literary and visual evidence on Ludovico's appreciation in Rome is brought to bear, in order to reassess his contemporary fame, superior to his cousins', as is suggested by works attributed to him in the main seventeenth-century Roman collections. His present-day partial disgrace is the result of a number of changes soon brought about by several factors, including the probability of untold doubts on his religious orthodoxy raising in the early seventeenth-century. His use of German prints especially in his late religious paintings may have a lot to do with this.
\end{abstract}

Malvasia non era originale, nel presentare Ludovico Carracci come il protagonista, l'ispiratore e il mentore, l'iniziatore della cosiddetta riforma carraccesca promossa, con vario accento ma sostanziale unità di intenti, dai tre cugini Carracci, anche tramite la loro accademia privata: ${ }^{1}$ l'avevano preceduto almeno il bolognese Giovan Battista Agucchi, ${ }^{2}$ il senese Giulio Mancini, ${ }^{3}$ il romano Giovanni Baglione ${ }^{4}$ e, sia pur di poco e ambiguamente, il tedesco Joachim von Sandrart. ${ }^{5}$ Il che significa semplicemente che Malvasia seguiva il mainstream storiografico peninsulare, non solo felsineo, secentesco, diffuso e sancito a livello internazionale da un tedesco di Norimberga competente e protestante: e poiché Agucchi era trapiantato a Roma e a Roma lavorava per Pietro Aldobrandini (tanto che per qualche moderno storico distratto può sembrare romano, come Mancini), ${ }^{6}$ non è dubbio che esprimesse, assieme ad

\footnotetext{
${ }^{1}$ Malvasia 1678, 354-515, specie 357-362 e 490-494.

${ }^{2}$ Vedi G.B. Agucchi, non solo per le parti del suo Trattato perduto trascritte a Roma da Mosini [= Massani] 1979, XXXVII-LXXIV passim (parzialmente riprese anche in Malvasia 1678, 379-380, 403-404, 449-450, 469-470, 471-473, 488-489), ma soprattutto nelle Lettere superstiti al Dolcini, allo stesso Ludovico, solo parzialmente pubblicate. Formalmente Ovidio Montalbani (Montalbani 1641 = Antonius Bumaldus 1641, 261-262) parla dei tre Carracci, senza mai nominarli individualmente, ma il suo esplicito rinvio a Dolcini 1631 (Dolcini 1631, 296-309: vedilo ristampato in Perini 1994, 85-104, specie 95-98, con commento parziale a 85-87) di fatto implicitamente sancisce la superiorità di Ludovico. ${ }^{3}$ Mancini, Salerno and Marucchi 1956, 216-220.

${ }^{4}$ Baglione 1642, 105-106.

${ }^{5}$ Sandrat J. von 1675-1680, II, 186-189 e, per la versione latina, Sandrat J. von 1683, 178-180, specie 180. Sulla problematicità della testimonianza di Sandrart, dipendente da una fonte romana che sbaglia molto sulla biografia dei Carracci, confondendo in parte Agostino e Ludovico, v. Perini Folesani 2015, 97-104.

${ }^{6}$ Pierguidi 2014, 68-78, specie 68.
} 
una propria opinione e propensione personale di gusto (condivisa con l'amico Bartolomeo Dolcini, devoto committente di Ludovico a Bologna), anche un pensiero circolante a Roma: tra i vari indizi che avvalorano questa tesi, ci sono l'invito dei Farnese a Ludovico per dipingere nel loro palazzo romano (sarà scelta di Ludovico mandarvi in sua vece Annibale e restare personalmente a Bologna), ${ }^{7}$ la testimonianza dell'archiatra pontificio Mancini e, appunto, la sintesi di Sandrart, che a Roma si era fermato a lungo e vi aveva formato le proprie conoscenze dell'arte italiana. ${ }^{8}$

Conferisce ulteriore sostegno alla tesi testé enunciata la presenza di quadri (veri o presunti) di Ludovico in diverse, ragguardevoli collezioni romane (non a caso sottolineata da Malvasia, ma affermatasi già qualche decennio prima, se perfino la Regina Cristina di Svezia e i Barberini credevano di possederne esemplari, ${ }^{9}$ dubbi e qualitativamente inferiori al sicuro Sebastiano Doria Pamphilj e ai quadri Pallavicini, Giustiniani, Sacchetti o Falconieri). ${ }^{10}$ A ciò si aggiungano i versi di Giovan Battista Marino pubblicati in onore postumo di Ludovico nella tarda Galleria (1620): ${ }^{11}$ benché il poeta napoletano abbia girovagato per l'Italia, è probabile che proprio a Roma, dove nel 1603 era al servizio di Pietro Aldobrandini (esattamente come Agucchi), si sia formato la sua idea di Ludovico, confortata poi dai suoi amici e colleghi bolognesi durante il successivo soggiorno emiliano-romagnolo, cui risalgono le sue due lettere superstiti a Ludovico. ${ }^{12}$

È Bellori che, con la sua nota indifferenza alla verità della storia e ai principi di una retta filologia, piegate entrambe alla sua visione politicamente partigiana e utilitaristica, ribalta tardivamente (e con successo) la narrazione, probabilmente per compiacere l'ambasciatore di Francia che, allora come ora, si trovava insediato a Palazzo Farnese, accidentalmente decorato da Annibale: ${ }^{13}$ la fortuna tuttora perdurante delle sue tesi fallaci e sempliciste è la migliore riprova della nozione comune che gli asini volano.

Malvasia, che per evitare "impegni" nel 1678 finge ancora di aspettare ansiosamente le $V i$ te belloriane (iuxta i principi cautelosi dell'onesta dissimulazione),${ }^{14}$ ma che in realtà si decide finalmente a scrivere di furia la propria Felsina Pittrice proprio perché le ha lette e vuol correggerne le menzogne e gli errori, dando contestualmente una lezione generale e aggiornata di metodo storiografico fuor di polemica, sottolinea con forza il primato di Ludovico (con-

\footnotetext{
${ }^{7}$ Come storicamente acclarato da Zapperi contro la testimonianza di Baglione: v. Zapperi 1986, 790-795.

${ }^{8}$ Per Mancini e Sandrart v. supra, rispettivamente note 3 e 5.

${ }^{9}$ Per Cristina di Svezia, v. Montanari 1997, 186-264, specie 240 (si tratterebbe del quadretto, invero assai dubbio, comprato sul mercato da una società ed esposto come autografo alla Pinacoteca Nazionale di Bologna: v. Bentini et al. 2006, 220-221, scheda n. 159); per i Barberini v. Aronberg Lavin 1975, 475-476.

${ }^{10}$ Per i quadri di Ludovico nella Galleria Doria Pamphilj, v. De Marchi 2016, 108-109, scheda FC 106 (San Sebastiano) e inoltre, parimenti autografo, la Madonna col Bambino e santi ibidem, p. 109, FC 198; per i quadri Pallavicini, v. Zeri 1959, pp. 78-79, n. 114; per i Giustinani, v. Danesi Squarzina 2003, 128-129, n. 70 e fig. 46 e inoltre 146-147, n. 67 e figg. 66-67; per i quadri passati per le Collezioni Sacchetti e Falconieri e oggi nella Pinacoteca Capitolina di Roma, v. Guarino, Masini 2006, 232-233, n. 100 (San Francesco in preghiera), 234-235, n. 101 (Testa di giovane, variamente attribuita ad Annibale o Ludovico), 236-237, n. 102 (Sacra Famiglia con San Francesco e Santa Caterina d'Alessandria), 238-239, n. 103 (Allegoria della Provvidenza), 240-241, n. 104 (Santa Cecilia), 242-243, n. 105 (Madonna col Bambino, nello stile di Ludovico, ma più probabilmente a mio giudizio Scuola).

${ }^{11}$ Marino 1979, 17 e 25.

${ }^{12}$ Ristampate in Perini 1990, 145-146, Appendice A.

${ }^{13}$ Su Bellori v. Perini Folesani 2014a, 127-138 (e, più in generale, tutti i saggi del volume, che integra gli studi raccolti in Bell, Willette 2002).

${ }^{14}$ In proposito v. Perini Folesani 2014b, 77-100, specie 80-82.
} 
vincendo agevolmente per secoli artisti di bella intelligenza e non comune talento come, nel Settecento, Charles de La Fosse, Fragonard e Sir Joshua Reynolds), ${ }^{15}$ ma, in polemica con Bellori e con il coté romano secentesco, aggiunge anche la notizia di una sostanziale avversione di Ludovico per Roma che è in realtà non solo un topos della sua polemica antivasariana, ${ }^{16}$ ma anche il riflesso, dal 1506 (anno della conquista militare di Bologna da parte di Giulio II), dell'atteggiamento prevalente nel ceto senatorio bolognese da cui egli stesso proviene (ogni storiografo racconta sé) e che solo in parte, probabilmente, corrisponde alla realtà artistica di Ludovico. ${ }^{17}$

È un fatto che la celebre pala della Conversione di Paolo un tempo in San Francesco a Bologna - e che, stando ad Arcangeli, avrebbe ispirato Caravaggio sulla via da Milano a Roma fu dipinta per l'altare di famiglia di un austero senatore e militare bolognese, Emilio Zambeccari, e pagata postumamente (1588) dal suo nipote, erede e successore nel seggio senatorio, Scipione Zambeccari, ${ }^{18}$ lo stesso che, da gonfaloniere (1589) avrebbe incaricato lo zio di Ludovico, Carlo Carracci, appassionato di matematica, di scrivere due memoriali tecnicamente ed esteticamente motivati per criticare il progetto di copertura della navata centrale di San Petronio iniziato dal Terribilia su incarico del Cardinal Legato Montalto, Cardinal nipote di Sisto V, ma la cui vera ragion d'essere era politica, determinata dal sentimento anticuriale della stragrande maggioranza del Senato felsineo. ${ }^{19}$ Certo è che i documenti prodotti da Carlo Carracci sortirono l'effetto desiderato di bloccare il progetto romano per vari decenni, specie dopo l'intervento del nuovo Papa Clemente VIII Aldobrandini, condizionando poi efficacemente la realizzazione compiuta da Girolamo Rainaldi solo verso la metà del secolo seguente: è vero quindi che la famiglia Carracci era effettivamente coinvolta nella battaglia politica anticuriale felsinea, a dispetto del fatto che, lungi dall'essere campioni di bolognesità quali li rappresenta Malvasia (Ludovico in primis), molti di loro (tra cui il ricco e potente Carlo, suo figlio Marcantonio, il di lui cugino Agostino e il padre di Ludovico, Vincenzo) prediligessero sottolineare le loro origini cremonesi, non solo esibendo nella firma il soprannome-toponimo "Cremona" o l'aggettivo Cremonese, ma abitando (è il caso di Ludovico) in una laterale di via Galliera, strada senatoria i cui dintorni ospitavano però gli artigiani lombardi immigrati a Bologna, nè forse è un caso che la sorella di Ludovico, Prudenza, si fosse sposata nel 1584 con il falegname Francesco Tacconi, abitante in un'altra laterale di via Galliera forse perché imparentato con una famiglia omonima di artigiani e pittori di Cremona (e forse anche per questo Innocenzo, suo primogenito nato nel matrimonio precedente, sarebbe stato pittore). ${ }^{20}$

\footnotetext{
${ }^{15}$ Vedi quanto già detto in Perini 1993b, rispettivamente 269-344, specie 280, 300, 315-318.

${ }^{16}$ Malvasia 1678, 359, 363-364, 403-404, 406, 436-437, 447, 466, 476-477, 480, 484, 490-494.

${ }^{17}$ Sull'importanza di Firenze (tradizionalmente negata dalla storiografia artistica bolognese) e di Roma nella formazione artistica e culturale di Ludovico v. quanto già sparsamente da me accennato in Perini Folesani 2010, 168-186, specie 183-185.

${ }^{18}$ Vedi la scheda di Feigenbaum 1993, 42-43, n. 19 (quasi tutte le schede del catalogo sono scritte dalla Feigenbaum).

${ }^{19}$ Dopo i miei primi studi (Perini Folesani 2010 e Perini Folesani 2013a, 110-126), v. ora Nicoletti 2017, 59-78, con importanti, preziose aggiunte e precisazioni di carattere storico e teorico sulla vicenda. Non mi risulta invece pubblicato l'intervento di Lisa Hillier "The "Gothic Tailor" from Bologna: Carlo Carracci and his Rivalry with Francesco Terribilia, al convegno Renaissance Conflict and Rivalries: Spheres, svoltosi a Bonn nel maggio 2014.

${ }^{20}$ Sulla famiglia Carracci, e in particolare su Carlo, v. intanto Perini Folesani 2016, 197-206, specie 200-201. Per il ritratto della Famiglia Tacconi di Ludovico, v. Bentini et al. (ed.) 2006, 236-237, n. 165 e, prima Feigenbaum 1985, $277-$ 278, n. 49.
} 
In questo contesto, la notizia, fornita da Malvasia, che Ludovico si sarebbe recato a Roma solo una volta in vita sua, tardivamente, nel 1602, per pochi giorni, giusto il tempo di controllare il lavoro eseguito da Annibale a Palazzo Farnese su sua ispirazione, di imporvi il suo sigillo di capobottega (apportandovi o meno correzioni marginali) ${ }^{21}$ e darsi una veloce occhiata in giro trova all'apparenza riscontro obiettivo in due ordini di prove ben note: quelle visive, fornite dalla sua Vocazione di Matteo, ${ }^{22}$ che rivela nella figura di Cristo il ricordo in controparte di quella del Caravaggio nel quadro romano di analogo soggetto, ma diversa invenzione e stile nella cappella Contarelli (la presenza, incongrua per la Palestina, di una colonna coclide tipicamente romana vuole forse rimarcare proprio l'ispirazione "romana" del quadro e la gara col Caravaggio, intesa in modo affatto opposto a quello, emulativo, suggerito al giovane Guido Reni dal Cavalier d'Arpino), ${ }^{23}$ e quelle documentarie, rese note da Heinrich Bodmer, che rivelano l'altro scopo (peraltro mancato) del suo viaggio a Roma, ovvero la richiesta al pontefice (Clemente VIII Aldobrandini) di fare dell'Accademia degli Incamminati o Desiderosi di Bologna una pubblica accademia, affiliata a quella romana di San Luca: proprio per questo Ludovico si sarebbe fatto accompagnare dal collega Denis Calvaert, il primo maestro di Guido Reni e di altri giovani illustri della bottega carraccesca. ${ }^{24}$

Tutto questo prova sì la realtà di quel suo viaggio a Roma, testimoniato anche dalla lettera al Brizio pubblicata nella Felsina Pittrice ${ }^{25}$ e da altre di Monsignor Agucchi al Dolcini che però allungano il suo soggiorno romano di circa un mese, ${ }^{26}$ ma non ci assicura affatto che questo sia stato l'unico, come vuole Malvasia: se non esiste traccia di ulteriori soggiorni posteriori (anzi, con l'aiuto della scuola Ludovico si organizza una sua Roma a Bologna, nel chiostro di San Michele in Bosco), v'è però la quasi certezza di almeno un soggiorno anteriore, presumibilmente anche di molti anni prima, indicativamente collocabile non solo prima del 1584 (affreschi di Palazzo Fava), ${ }^{27}$ ma forse addirittura prima del 1578 - anno di iscrizione di Ludovico ventitreenne come maestro alla compagnia felsinea dei pittori, compresa nell'arte dei Bombasari: ${ }^{28}$ viaggio dunque coincidente con il pontificato del bolognese Gregorio XIII Boncompagni. Se la Sacra Famiglia di Nantes fosse davvero un incunabolo della produzione artistica di Ludovico giovanissimo (del che si può legittimamente dubitare, nonostante la caotica congerie di testimonianze visive problematiche e mal conciliabili sui suoi esordi: di certo non può essere di Annibale), ${ }^{29}$ la sua cruda durezza disegnativa accompagnata da un chiaroscuro molto marcato ne farebbe una testimonianza forte di un approccio più romano (anche per la curiosa aura transalpina, e dunque internazionale, di certi brani) che to-

\footnotetext{
${ }^{21}$ Malvasia 1678, 406.

${ }^{22}$ Bentini et al. (ed) 2006, 228-229, n. 163.

${ }^{23}$ In proposito v. Perini 2002, 303-311.

${ }^{24}$ Perini 1990, 83-84 (con bibliografia precedente).

${ }^{25}$ La trascrizione autografa di Malvasia sopravvive nelle carte Hercolani di provenienza Malvasia: BCB, ms B 153, n. 11: cfr. Perini 1990, 110-111 (con elenco delle trascrizioni precedenti).

${ }^{26}$ Cfr. Stanzani 1993, 199-268, specie 234-235.

${ }^{27}$ Per le fonti iconografiche classiche e moderne, felsinee ed extra felsinee, delle divinità a chiaroscuro della Sala di Giasone, v. Perini 2006, 189-211 (con bibliografia precedente) e, successivamente, Emiliani 2011.

${ }^{28}$ L'iscrizione a maestro dell'arte certificava infatti, tra l'altro, il completamento dell'iter formativo, che all'epoca non poteva non includere uno stage di studio/lavoro nelle capitali della Maniera, ovvero Firenze e Roma.

${ }^{29}$ Sulla questione attributiva, v. Benati 2006, 126-133, specie 133, nota 42 e fig.n.n. a p. 130, con ricapitolazione delle vicende attributive, ove si contrappongono la fazione pro-Ludovico (in cui spesseggiano gli studiosi statunitensi e in cui io mi riconosco agevolmente) e quella pro Annibale (ove milita lo stesso Benati, con scarso seguito, a parte Schleier).
} 
scano o bolognese, e più michelangiolista che raffaellista. (La citazione raffaellesca nel tardo Sogno di Giacobbe, se contribuisce a garantire un'ovvia attrazione per la lezione romana di Raffaello, nelle Logge, non vale a confermare un viaggio giovanile a Roma, dato che il quadro, comunque posteriore al viaggio del 1602, è all'incirca coevo alla traduzione grafica delle Logge per opera di Lanfranco e Badalocchio, pubblicata nel 1607, e che essa non conteneva il primo intaglio di quella scena specifica). ${ }^{30}$

Dalla nascita Ludovico, col fratello Vittorio, era stato iscritto dal padre Vincenzo all'arte dei beccai di cui questi era obbediente (mai ne fu maestro): ${ }^{31}$ a Bologna era un'arte prestigiosa e potente, i cui maestri (i Canobi, ad esempio) erano molto ricchi e rispettati. L'iscrizione alle arti (a seconda dei tempi e delle arti in questione) a Bologna era un dovere non solo sociale, ma economico e previdenziale: ${ }^{32}$ Vincenzo aveva prenotato quindi ai figli piccolissimi un lavoro e un posto sicuri, caso mai non avessero avuto altri talenti, sottoscrivendo così una sorta di assicurazione per il loro futuro. Ancor più ricca e prestigiosa, però, era l'Arte dei Drappieri e Strazzaroli cui apparteneva il fratello di Vincenzo, Carlo, che, come maestro, vi ricoprì più volte, fino alla morte occorsa nel 1600 , la carica di massaro: ${ }^{33}$ alla sua morte gli subentrò il figlio Marcantonio, notaio regolarmente iscritto alla propria arte e che, in barba alle leggi che vietavano la doppia iscrizione, sedette anche nel Consiglio dei Drappieri. ${ }^{34}$ Del resto ai Drappieri e Strazzaroli appartenevano i cadetti di varie famiglie importanti di Bologna, tra cui, oltre ai Paleotti della stessa famiglia dell'arcivescovo, anche i Fava primi committenti dei tre Carracci esordienti. Solo il padre di Agostino e Annibale, Antonio, era iscritto ad un'arte secondaria e poco stimata, quella dei sarti, ${ }^{35}$ e non vi ebbe nemmeno vita facile, come ha dimostrato Zapperi: ${ }^{36}$ c'è poco da dubitare, quindi, che fosse Carlo - non Antonio l'artefice delle fortune non solo dei suoi discendenti diretti, ma anche di quelli dei suoi fratelli Vincenzo e Antonio, introducendo (non sempre con successo) i tre giovani pittori ai suoi colleghi Fava, Zambeccari, Gessi, non meno che allo studio della matematica, della prospettiva, dell'astronomia, ${ }^{37}$ delle quali si dilettava personalmente, potendo vantare una congrua

\footnotetext{
${ }^{30}$ Per il quadro di Ludovico, v. Emiliani1993. 137-138, n. 63 e Bentini et al. 2006, II, 270-272, n. 180. Per il dipinto di Raffaello e le sue riproduzioni, v. Bernini Pezzini, Massari and Prosperi Valenti Rodinò 1985, 72-112, specie 73, n. 12 (la stampa di Jacob Bos, $\uparrow 1580$, è la più antica e di modesta qualità artistica), e, per la Serie delle Storie del Testamento Vecchio, di Sisto Badalocchio e Giovanni Lanfranco (1607), con dedica ad Annibale, v. pp. 77-80, nn. 1-51, specie 78, n. 21 (opera di Lanfranco). La serie fu poi copiata anche da Galanino, ma in controparte nel 1615 (ibidem, pp. 80-81, nn. 146, specie 19).

${ }^{31}$ Vedi Fanti 1980, 158-160 e 163, nota 95.

${ }^{32}$ Sulle arti a Bologna v. il fondamentale studio di Gheza Fabbri 1988. Per il ruolo di spicco dell'Arte dei Beccai in età rinascimentale a Bologna (a differenza di quanto era avvenuto in età medievale e di quanto accadeva in generale in Italia) v. ibidem, pp. 26-28 e 49-51, nonché passim.

${ }^{33}$ Su questa arte, v. Gheza Fabbri 1988, 109-132 e passim e, più specificamente su Carlo Carracci, v. i riferimenti dati a note 19 e 20.

${ }^{34}$ Le carte dell'Arte dei Drappieri e Strazzaroli, inclusi i documenti relativi al Consiglio, a differenza di quelle di altre corporazioni bolognesi, non sono conservate presso l'Archivio di Stato di Bologna, bensì costituiscono un Fondo speciale della sezione manoscritti della Biblioteca dell'Archiginnasio. Le affermazioni che si fanno in questo saggio relativamente ai rapporti tra i Carracci (specie Carlo e il figlio Marcantonio) e questa Arte sono basate su una ricognizione sistematica di queste carte avviata negli scorsi anni.

${ }^{35}$ Cfr. Gheza Fabbri 1988, 28-29 Antonio però aveva sposato una Zenzanini, esponente di una famiglia influente nell'Arte dei Drappieri e Strazzaroli (in proposito v. nota seguente).

${ }^{36}$ Zapperi 1989, specie pp. 5-20 (anche per i molteplici rapporti matrimoniali tra i Carracci e gli Zenzanini).

${ }^{37}$ Vedi supra, nota 33.
} 
biblioteca specializzata di oltre 280 volumi. ${ }^{38}$ Parimenti non può essere casuale la scelta di Carlo di far dipingere il camino di casa propria non ad Agostino, non ad Annibale, ma proprio a Ludovico, ${ }^{39}$ il più manifestamente interessato dei suoi nipoti, fin dal debutto, alle applicazioni della prospettiva alla pittura. ${ }^{40}$ (E l'invenzione di Ercole vittorioso pensata privatamente da Ludovico per un familiare nel 1594 diventa subito dopo a Roma, grazie all' Ercole al bivio nel soffitto del Camerino Farnese che lo riprende in controparte, il marchio romano della ditta Carracci). ${ }^{41}$

Orbene, la serie di monocromi con le effigi degli dei antichi che scandiscono le scene del fregio della Sala di Giasone a Palazzo Fava hanno fonti iconografiche classiche e moderne che ho già individuato almeno parzialmente in altra sede, molti anni fa: ${ }^{42}$ tra le moderne, spiccano il sansovinesco Nettuno del Palazzo Ducale di Venezia (trasformato in Eolo) e il Bacco di Michelangelo, che prima di arrivare a Firenze in data imprecisata (tra il 1572 e il 1591), era a Roma, tra casa Galli e Villa Medici. Tra le statue antiche, anche il Saturno è una libera ripresa del Sileno con Bacco bambino del Louvre, all'epoca forse ancora in collezione Muti a Roma, essendo attestata in collezione Borghese solo a partire dal 1613, e proprio come Saturno. ${ }^{43}$

Venezia (come Parma) è tappa riconosciuta dello "studioso corso" carraccesco: non così Firenze e Roma, con la differenza che per Firenze, checchè se ne dica oggi a Bologna, esistono - almeno per Ludovico ed Agostino - prove certe e indizi cogenti, in parte noti e accennati dallo stesso Malvasia, in parte ricostruibili a posteriori. ${ }^{44}$ È Malvasia, per esempio, a riferire non solo negli appunti di una attività giovanile di Ludovico con il Passignano, a Firenze: ${ }^{45}$ ma con il coetaneo toscano Ludovico poteva al massimo collaborare, come sottoposto al gran Federico Zuccari, nel cantiere della decorazione della cupola di Santa Maria del Fiore (15751579), ${ }^{46}$ ed è tra Firenze e Urbino, tra lo Zuccari e Barocci (che Ludovico può aver incontrato a Firenze, se non lo aveva già conosciuto prima) che si svolge una parte rilevante del suo percorso formativo. ${ }^{47}$ Di Barocci si è ben accorto Andrea Emiliani (pur escludendo a priori, credo troppo frettolosamente, una visita di Ludovico all'erta e scomoda Urbino, optando per una gita piana nella più vicina - e malarica - Ravenna, dove però non avrebbe potuto vedere tutto quel che gli serviva); ${ }^{48}$ di Zuccari, invece, ognuno tace.

\footnotetext{
${ }^{38}$ Lisa Hillier, abstract dell'intervento 'Reading Continually the Great and Ancient Authors': The Mathematical Library of Carlo Carracci, al workshop Printing Mathematics in the Early Modern World, svoltosi ad All Souls College, Oxford nel dicembre 2014 (non mi risulta che il testo sia stato pubblicato).

${ }^{39}$ Feigenboum 1985, 305-308, n. 69.

${ }^{40} \mathrm{Su}$ Ludovico e il suo interesse per la prospettiva, v. Perini Folesani 2013a, 111-115.

${ }^{41}$ Ibidem, 123.

${ }^{42}$ Perini 2006.

${ }^{43}$ Haskell, Penny 1982, 306-307, n. 77.

${ }^{44}$ Vedi almeno la bibliografia alle note 17 e 19.

${ }^{45}$ Malvasia 1678, I, 359.

${ }^{46}$ Zapperi 1989, 15 e Perini 1990, 50, nota 55. Sulla decorazione della cupola del Duomo, v. Acidini Luchinat 1999, II, 65-103.

${ }^{47}$ Vedi quanto delineato in Perini Folesani 2013b, 3-43, specie 6-15.

${ }^{48}$ Emiliani 1984, XVII-LXX, specie XLII e LIII-LV. Il criterio su richiamato della conveniente accessibilità vale anche, evidentemente, per il Casino di Pio IV o l'Appartamento del Belvedere a Roma.
} 
Eppure è proprio dal coté zuccaresco che viene a Ludovico una delle cifre più amate $\mathrm{e}$ insistite del suo repertorio linguistico, la figura a losanga: ${ }^{49}$ vero è che poteva trovarne un esempio felsineo (un vero unicum) anche nelle due figure dell'Inverno e dell'Autunno nell'impresa dell' Accademia dei Gelati, opera del suo maestro Prospero Fontana (cui lo legava una reciproca incomprensione), ${ }^{50}$ e che tanti poteva rintracciarne nella cupola del duomo fiorentino, ma solo a Roma, nella cappella Frangipane di San Marcello al Corso (opera del fratello maggiore di Federico, Taddeo) poteva vedere l'abbinamento di quella figura con altra (parimenti angelica e obliqua) che avrebbe egli stesso adottato e reimpiegato, almeno una volta, nell'Orazione dell'Orto della National Gallery di Londra, e da cui sarebbe partito per numerose variazioni sul tema nei tardi affreschi di Piacenza, ${ }^{51}$ quando ancora corrispondeva col vecchio Federico Zuccari, cui quello stesso schema era parimenti caro. (̇̀ appena il caso di notare che ricorda assai da vicino il cosiddetto Gladiatore Borghese di Agasia, peraltro dissotterrato solo più tardi, nel 1611). ${ }^{52}$ Vero è che, frequentando Federico Zuccari a Firenze, Ludovico poteva aver visto i cartoni o gli schizzi preparatori delle opere sue e del fratello, senza dover postulare per forza una gita a Roma prima del 1602: ma altri dipinti di Ludovico raccontano, con la forza delle immagini, un'altra storia.

Per Bartolomeo Dolcini Ludovico dipinge una serie di quadri (solo in parte sopravvissuti, ma quasi tutti noti almeno come invenzione, tramite disegni preparatori o copie di bottega: manca solo - forse non per caso - l'Agostino d'Ippona). ${ }^{53}$ Tra essi rifulge l'Abramo e $i$ tre angeli della Pinacoteca Nazionale di Bologna. ${ }^{54} \mathrm{Se}$, come ho già dimostrato a Mosca, due dei tre angeli rivelano la sua precisa conoscenza e deliberata allusione ad una qualche copia greco-balcanica (cioè veneziana) della iconica Trinità di Andrei Rubliov, l'Abramo riprende invece con attenzione quasi archeologica il tipo del prigioniero dace e non so quanti ne potesse trovare fuori Roma (certo ce n'erano a Villa Medici, ai lati della Loggia sul Giardino). ${ }^{55}$ Ma perché usare quel bizzarro costume antico ed esotico, sì, ma continentale, europeo, per un patriarca veterotestamentario di provenienza mediorientale? Forse perché l'aveva già fatto Michelangelo nel suo celeberrimo Mosè, che Ludovico cita puntualmente, traducendolo in cifra zuccaresca (la losanga), nella sua Trasfigurazione del 1595/96, così amata, oltre che da Reynolds, da Benjamin West (FIG. 1). ${ }^{56}$ Sicuramente ci saranno state stampe di traduzione del Mosè e, soprattutto, modellini plastici, ma non so quanti potessero suggerirgli la deformazione compositiva cui lo sottopone Ludovico, più di una visione diretta, opportunamente angolata, che certamente l'avrebbe facilitata.

E che dire poi dell'Isaia affrescato nella Cappella Gessi della Madonna della Pioggia a Bologna (1585 circa), danneggiata dalle bombe della II guerra mondiale ? ${ }^{57}$ Di là dal costume

\footnotetext{
${ }^{49}$ In proposito v. Perini Folesani 2013b, 38-39, nota 104.

${ }^{50}$ Sul quadro di P. Fontana v. ad es. Fortunati 1994, 177, scheda n. 27 e tav. a colori. Sui difficili rapporti tra Prospero e Ludovico, v. Malvasia 1678, I, 358-360.

${ }^{51}$ Per gli spunti zuccareschi, v. supra, nota 49.

${ }^{52}$ Haskell, Penny 1982, 221-224, n. 43.

${ }^{53}$ Sulla serie dei quadri Dolcini v. l'intervento ricapitolativo di Bianchi 2014, 145-158, e qualche precisazione ulteriore, relativa a quelli di cui sopravvivono disegni preparatori, in Perini Folesani 2016, 203, note 48-49.

${ }^{54}$ Bentini et al. 2006, II, 268-269, n. 178.

${ }^{55}$ Perini Folesani 2020a, 35 e 2020b, 155-164.

${ }^{56}$ Bentini et al. 2006, 249-250, n. 170. Sulla fortuna del quadro, v. Perini 1993b, 320.

${ }^{57}$ Vedilo ottimamente riprodotto in Bodmer 1939 e cfr. Feigenbaum 1985, 228-232, n. 23.
} 
dace ormai acquisito come ebraico, la sua posa meditabonda rinvia a quella di un altro profeta affrescato a Roma, nell'arco di ingresso della Cappella Pucci a Trinità dei Monti, quello di Perin del Vaga, che non penso godesse di altrettanta fortuna riproduttiva quanto il Mosè michelangiolesco (ma che, in seguito, avrebbe colpito ancor più vivamente Reynolds). ${ }^{58} \mathrm{La}$ figura di Perino risulta innaturalmente stirata, allungata, per conformarsi, con quella del compagno, all'arcata gotica della chiesa che decora: ed ecco che con ghiribizzo innovativo Ludovico, invece di stirarla ulteriormente, contro le sue abitudini apparenti, la ricompatta, anche per adattarla allo spazio angusto della sua cappella. Si direbbe che parte del suo gioco (ri)creativo sia la reinterpretazione ipercorrettiva del modello illustre, deformando per schematizzazione geometrica forzosa e contraria l'invenzione acquisita di partenza.

D'altro canto, il modo in cui siede Isaia, l'incrocio delle sue gambe, ritorna in tanti altri dipinti di Ludovico (ad esempio il Cristo nella Visione della Porziuncola di Madrid: FIG. 2) ${ }^{59}$ e ricorda anche quello di un altro quadro perduto di Ludovico, il Girolamo di collezione Monti a Bologna, sparito in età napoleonica, poco dopo esser stato tradotto malamente a stampa (ma, ancora una volta, è un disegno di Reynolds a confortarne la sostanziale attendibilità riproduttiva). ${ }^{60}$ Qui però il movimento della figura si complica, per la torsione in senso contrario del busto che determina una tensione compositiva creata dallo schema della losanga scomposta in due triangoli speculari uniti per un angolo. Tra i dipinti superstiti che ne recano traccia si possono ricordare, almeno, la Madonna già Giustiniani, ora al Metropolitan (FIG. 3), e il Pilato travestito da sultano che se ne lava le mani nell'affresco con l'Ecce Homo già nel Palazzo un tempo Hercolani di Via Galliera. ${ }^{61}$ La fonte di questo schema, però, è, ancora una volta, zuccaresca: lo si ritrova infatti a Roma nell'affresco del profeta con Sibilla sulla parete dell'Oratorio del Gonfalone, ${ }^{62}$ o nel San Marco di Santa Caterina de' Funari, ${ }^{63}$ o in qualche Madonna in sacra conversazione (la Pala Zuccari di Sant'Angelo in Vado, ad esempio), ${ }^{64}$ o figura allegorica di scuola.

Certo non è da Federico Zuccari, però, che la riprende Guercino nel giovanile Elia nutrito dai corvi: ${ }^{65}$ e di nuovo, il Girolamo guercinesco che sigilla lettere a Palazzo Barberini (di cui esistono più versioni e copie) sicuramente ripensa spunti ludovichiani bolognesi e piacentini, che dubito sarebbero esistiti senza qualche esempio tosco-romano precedente. ${ }^{66}$ La vitalità dei rapporti, biunivoci, del dialogo tra Ludovico da un lato, i tardo-manieristi tosco-romani dall'altro si tocca con mano confrontando i suoi profeti della Cappella Gessi con quelli dipin-

\footnotetext{
58 Vedi l'affresco in Parma 1997, 55-62 e fig. 47 e 258-260, scheda n. A.VI e, per lo schizzo fattone da Reynolds nel taccuino di Plymouth, Perini Folesani 2020c, 199-200.

${ }^{59}$ Vedi Feigenbaum 1985, 376-377, n. 108.

${ }^{60}$ Vedi Perini 1993b, 315 e 317, figg. 32-33.

${ }^{61}$ Rispettivamente in Feigenbaum 1985, 416-417, n. 121 (Madonna al Metropolitan Museum) e 300-302, n. 64 (Ecce Homo).

${ }^{62}$ Vedine un'eccellente riproduzione a colori in Bernardini 2002, fig. 6 a.

${ }^{63}$ Acidini Luchinat 1999, II, 44, fig. 3.

${ }^{64}$ Acidini Luchinat 1999, II, pp. 232-234, fig. 1.

${ }^{65}$ Salerno 1988, 147, n. 68.

${ }^{66}$ Per il quadro di Guercino, v. Salerno 1988, 116-117, n. 36. Per figure di Ludovico in pose in qualche modo rapportabili, v. ad es. la posa del crocifero a sinistra nella pala per la Cappella Montecalvi al Corpus Domini di Bologna (Emiliani 1993, 131-132, n. 60), nell'angelo che suona il triangolo nel Paradiso di San Paolo (Emiliani 1993, 167-168, n. 76), alcuni dei telamoni monocromi del Chiostro di San Michele in Bosco (Emiliani 1993, fig a p. CIX, ripetuta a p. 178), gli angeli del sottarco del Duomo di Piacenza (Emiliani 1993, fig. a p. XLVI).
} 
ti, circa trent'anni dopo, da Antonio Circignani, il Pomarancio in Santa Maria in Traspontina a Roma. ${ }^{67}$ Una coincidenza?

Ma se il problema artistico iniziale di Ludovico era di riformare la tradizione manierista tosco-romana arricchendola con la ripresa di una ricerca realistica, ispirata a suggestioni lombardo-venete e suggerimenti culturali di più ampio raggio (come dimostra l'attenzione, paradossale, all'iconografia postbizantina, secondo una tradizione padana che risale già a Savoldo), ${ }^{68}$ allora stupisce meno, nella ricerca dell'esotico, l'attenzione di Ludovico all'arte tedesca, di Dürer (forse anche tramite i falsi di Marcantonio Raimondi), Cranach e non solo. Agli esempi già individuati, non per caso, da studiosi germanici ${ }^{69}$ si potranno aggiungere qui la perduta tela della Cappella Gessi con 1'Adorazione dei Magi (1590 circa), ${ }^{70}$ che ha la stessa fonte di Crevalcore usata con più libertà in controparte, nonché 1'Immacolata per la Madonna degli Scalzi (FIG. 4) di struttura latamente teutonica, o l'invenzione per la perduta Estasi di Paolo già Dolcini, ${ }^{71}$ recuperata da una stampa di Schongauer con le Tentazioni di Antonio, ${ }^{72}$ sostituendo arditamente gli angeli ai diavoli, o, infine il Sebastiano di Gravina di Puglia, che per metà riprende un'invenzione di Schongauer ritradotta da Israel van Meckenem. ${ }^{73}$ Sono tutti esempi abbastanza cogenti, rinforzati dalla problematica Crocifissione di Ferrara, la cui evidente impostazione grünewaldiana si accoppia ad una scelta iconografica (il Crocefisso tra i Padri dell'Antico Testamento al Limbo, il cui tema è - in sintonia con i Dogmenbilder di Cranach - la salvezza per fede, non tramite le opere) certamente innovativa (e dunque, già per questo sospetta) e perciò di ortodossia cattolica non così pacifica. ${ }^{74}$ Che le parole non siano vuote, ma siano portatrici di senso e per di più di un senso incerto, passibile di ambiguità interpretative, non è scoperta pirandelliana, sull'onda di acquisizioni filosofiche germaniche posthegeliane: è la preoccupazione evidente e ossessiva di Gabriele Paleotti, nel suo Discorso intorno alle immagini sacre e profane (1582), il cui libro I, fondato sui dogmi della patristica anche greca, e sulla lezione semiotica della Scolastica, sviscera il tema della significazione, considerando l'immagine equiparabile alla parola in quanto segno significante, ed anzi ad essa superiore, in quanto dotata di una (presunta) universalità naturale che supera e trascende le barriere linguistiche e culturali. ${ }^{75}$ (L'esperienza missionaria extraeuropea dei gesuiti avrebbe ben presto sconvolto con la cogenza dei fatti, cioè delle reazioni ostili alle immagini cattoliche altrimenti interpretate, l'ottimismo ingenuo dell'assunto). ${ }^{76}$

\footnotetext{
${ }^{67}$ Per gli affreschi in Santa Maria in Transpontina, realizzati verosimilmente tra il 1616 e il 1618, v. tra i contributi più recenti Barroero 1983, 1-16, specie 10; Giffi 1983, 17-30, specie 28-29; Freeman Bauer 1983, 31-34, specie 32 e 33, fig. 3; Barroero 1984, II, 513-523.

${ }^{68}$ Per l'attenzione di Savoldo ai prototipi bizantini v. Perini Folesani 2020a, e Perini Folesani 2020b, 158-160, nonché, indipendentemente, Casciello 2018, 245-281.

${ }^{69}$ Vedi Hermann Fiore 2007, 341, n. VII.15 e Keazor, 2007, 185-187.

${ }^{70}$ Vedilo ben riprodotto in Bodmer 1939, Ludovico Carracci.

${ }^{71}$ Vedine il disegno preparatorio in Bohn 2004, 308, scheda n. 163.

${ }^{72}$ Per questa stampa v. Campbell Hutchinson 1996, 174-179, n. 801.054.

${ }^{73}$ Cfr. per il quadro di Ludovico, la scheda in Benati, Paolucci (eds.) 2008, 112-113, scheda n. 1 e Faietti and Hernad 1993, 122 e 386, scheda e fig. n. 244. La stampa di Israhel van Meckenem riprende in controparte un'invenzione di Martin Schongauer (1490): v. Campbell Hutchinson 1996.

${ }^{74}$ In proposito v. Perini 2007, 156-167 e più recentemente Perini Folesani 2016, 197-199 e 202-204.

75 Vedilo ristampato in Barocchi 1960-1962, II, 117-509, specie 209-211 e passim.

${ }^{76}$ Vedi i rinvii forniti in Perini 2007, 132-133.
} 
In ogni caso, le scelte iconografiche, le citazioni visive - soprattutto in un contesto religioso cinquecentesco di contrapposizione violenta tra chiesa cattolica e Riforma (a Roma sussunta nell'etichetta generica - non specifica - di luteranesimo) non sono, non erano anodine o irrilevanti, anche se la loro valutazione dipendeva molto dalla cultura, sensibilità e intelligenza - tutte molto aleatorie - dell'Inquisitore: le note vicende di Paolo Veronese (1573) per 1'Ultima Cena di San Zanipolo, poi trasformata in Convito a Casa Levi, lo dimostrano con chiarezza. ${ }^{77}$ Non è quindi pensabile che Ludovico Carracci utilizzasse senza consapevolezza o con indifferenza immagini semanticamente marcate: le verità dogmatiche possono essere svuotate, arricchite o deviate da scelte linguistiche preordinate e la svolta antigraziosa, austera, dura, scabra ed essenziale, ermetica ed intimista di Ludovico tardo vorrà pur dire qualcosa. Lo dimostra, nel 1616, la sua deliberata riscrittura del Martirio di Santa Margherita (1611) dello Scarsellino ferrarese, che proprio dalla scuola carraccesca indirettamente discendeva e che l'aveva dipinto per la stessa committente di Ludovico (Margherita Gonzaga). ${ }^{78}$

È vero che il cattolicesimo è etimologicamente onnicomprensivo e con un'acclarata, bimillenaria capacità di assorbire, riciclare e reintegrare qualsiasi devianza (o quasi): onde il Sebastiano di Gravina di Puglia (1599), frutto imprevedibile di una rielaborazione trasognata e paradossalmente graziosa di una stampa germanica, già di per sé sospetta, ${ }^{79}$ ma destinato a una fruizione privata, ${ }^{80}$ diventa però, un quarto di secolo più tardi perfettamente accettato su un altare cattolico, domenicano per di più!, grazie alla ripresa in una pala imponente di Guido Cagnacci e vien diffuso vieppiù dalla variante creata a Roma da Annibale Carracci (e/o da Albani) oggi a Quimper, a Genova e a Forlì. ${ }^{81}$ Ma altro è un altare di una chiesa della periferica e un po' inquieta Legazione di Romagna, altro è un altare di una chiesa romana, anzi, della Chiesa di Roma per eccellenza, San Pietro. La nota vicenda della mancata commissione di una pala in quella chiesa, certamente ricercata da Ludovico col sostegno di Agucchi (1603) e certamente rifiutata dal responsabile della committenza, il cardinal Baronio, che a Ludovico preferì - non senza inconsapevole ironia - l'antico compagno di studi toscani di Ludovico, Domenico Passignano, ${ }^{82}$ non è il segno di un fallimento artistico, di un'insoddisfazione estetica, o di un'inadeguatezza formale, ma - pur nell'ambito dei sempre oscuri maneggi di Curia, non insensibili alle esigenze politiche e alle pressioni personali - il sospetto (nulla più che un sospetto: altrimenti ci sarebbero stati provvedimenti espliciti) di un'inadeguatezza dogmatica per quel ruolo.

Lo svagato Sogno di santa Caterina a Washington (FIG. 5), ${ }^{83}$ se è il quadro regalato dal pittore ad Agucchi per questa circostanza o per propiziare il riconoscimento ufficiale

\footnotetext{
${ }^{77}$ In proposito v. ibidem, 172-174 e Grasman 2009, 125-134 (con bibliografia precedente).

${ }^{78}$ Per la pala dello Scarsellino (1611), v. Novelli 2008, 298, scheda n. 48 e fig. a p. 67 (con riferimento alla pala di analogo soggetto del Veronese a Brescia; in precedenza aveva sottolineato anche le somiglianze con quella di Ludovico); per quella di Ludovico, v. in Emiliani 1993, 163-166, n. 75.

${ }^{79}$ Per la definizione di "pittura sospetta" v. Paleotti, in Barocchi 1960-1962, II, 276-279 e passim.

${ }^{80}$ Probabilmente è il quadro in collezione Ginetti, già Rinaldi, per cui v. Stanzani 1993, 226.

${ }^{81}$ Per il quadro di Guido Cagnacci, v. Benati, Bona Castellotti 1993, 56-59, n. 1. Per quello attribuito ad Annibale, con sue repliche/copie, v. Puglisi 1999, 103-104, scheda n. 22a e 22.v.a, fig. 41.

${ }^{82}$ Sulla vicenda, v. Perini Folesani 2016, 204-205. Si noti incidentalmente che il Passignano (i cui rapporti giovanili con Ludovico sono accennati supra, nota 45) era stato indicato da Agucchi come referente per la qualità artistica di Ludovico! (cfr. Stanzani 1993, 238).

${ }^{83}$ Emiliani (ed.) 1993, 70-72, n. 33.
} 
dell'Accademia carraccesca a Roma ${ }^{84}$ così come i trasognati protagonisti di vari martíri padani che sembrano tradurre l'autenticità del dramma agiografico in una sorta di cupa favola a passo di danza sul palcoscenico di un teatro di per sé luogo di finzione, non di verità, erano imbarazzanti (non solo per le possibili evocazioni dei danzanti manigoldi düreriani che avevano già attratto Jacopo Bassano): ${ }^{85}$ ma legare l'imbarazzo a ragioni stilistiche piuttosto che semantiche o semiotiche, per non dir dogmatiche, è tutto sommato elegante e rassicurante, benché fuorviante. Le omissioni, le divagazioni o le mere elencazioni prive di commento di queste tele da parte di Malvasia storiografo, ma anche teologo (in odore di giansenismo) sono la riprova di un problema obiettivo, a un livello che poteva sfuggire a molti, Bellori compreso, ma più difficilmente a lui che, da bravo giurista, ricorreva perciò ad una tecnica di difesa standard (tacere il superfluo, il discutibile, il pericoloso). ${ }^{86}$

La sfortuna critica di Ludovico comincia probabilmente da qui: dalla necessità di non affrontare un percorso critico e storico di verità, rispettando per forza una sua scelta essenzialmente nicodemita, e quindi dall'impossibilità di enunciare e far capire le sue vere motivazioni artistiche, compresa l'adozione simultanea di modi diversi ${ }^{87}$ Si tolgono così punti di riferimento critici essenziali. Ridurre il discorso su Ludovico a un livello meramente stilistico porta al generoso ma deviante riscatto espressionista operato da Francesco Arcangeli, ${ }^{88}$ all'idea troppo limitativa di un suo tardo ripiegamento interiore che favorirebbe committenze marginali e provinciali (che tali non sono). I successivi, inani giochetti attribuzionistici postvolpiani di cui il povero Ludovico è fatto frequentemente oggetto derivano inconsapevolmente da questa forzata deprivazione di senso. È la strada che consente a Bellori di promettere una biografia di Ludovico, per non stenderla mai e di favorire anche per questa via la fortuna alternativa di Annibale e Agostino, sicuramente più facili e gradevoli e già all'apparenza assai meno problematici, ma solo perché meno innovativi, originali, densi e - nonostante tutto- coraggiosi. ${ }^{89}$ E siccome Roma è il luogo delle fortune facili ed effimere, all'ombra di un potere transeunte e superbo fondato sul compromesso che sembra garantirgli una supposta eternità, Ludovico a Roma era destinato a sparire, dapprima dalle pareti delle collezioni (con sostituzioni attributive più o meno motivate e alienazioni) e poi dalla memoria storica, iuvante Bellori. Nel secolo scorso il longhismo ha trasferito con successo l'operazione a Bologna, dove, addirittura, gli è stata rubata l'immagine, facendo del suo autoritratto con padre, zio (presumibilmente Carlo) e fratello Paolo un supposto autoritratto di Annibale, con papà e nipote omonimo, Antonio (che però non era neanche nato) e uno zio o committente a scelta. ${ }^{90}$

Giovanna Perini Folesani

Università degli studi di Urbino Carlo Bo

giovanna.perini@uniurb.it

\footnotetext{
${ }^{84}$ Sul quadro donato all'Agucchi, v. Stanzani 1993, 238, lettera di Agucchi a Baronio (1603, come realizzata nel 1602).

${ }^{85}$ Per i quadri di martiri di Ludovico v., oltre a nota 78, anche in Emiliani (ed.) 1993, 171-173, nn. 78. Per le note tangenze tra Jacopo Bassano e Dürer, si confronti il carnefice di spalle nella Decapitazione del Battista di Jacopo Bassano a Copenhagen con i due esempi düreriani presenti rispettivamente nella stampa di Cristo caduto sotto la croce dalla Grande Passione e in quella del Martirio di Santa Caterina: cfr. Fara 2007, 228.

${ }^{86}$ Su Malvasia giurista v. Agnati 2012, 55-72 e Agnati 2014, 399-451 e inoltre Perini 2010-2011, 312-327.

${ }^{87} \mathrm{Su}$ Ludovico e la teoria musicale, v. Perini Folesani 2013a, 121-122.

${ }^{88}$ Vedi, in particolare, Arcangeli 1970, specie 38-45 e 181-223.

${ }^{89}$ Sulla mancata biografia ludovichiana di Bellori, v. Perini 1993b, 286-287.

${ }^{90}$ Sulla questione dell'Autoritratto di Brera, v. Perini 1993a, 355-385, e, più recentemente, Perini Folesani 2021, e Perini Folesani 2014b, 79-80.
} 


\section{BIBLIOGRAPHY}

Acidini Luchinat C. 1999: Taddeo e Federico Zuccari, fratelli pittori del Cinquecento, Milano-Roma, II.

Agnati U. 2012: "Malvasia giurista. Dati e ipotesi per un profilo", in S. Frommel (a cura di), Crocevia $e$ capitale della migrazione artistica: forestieri a $\mathrm{Bo}$ logna e bolognesi nel mondo (secolo XVII), Bologna, 55-72.

Agnati U. 2014: "La biblioteca giuridica di Carlo Cesare Malvasia”, Studi urbinati - A - Scienze giuridiche, politiche ed economiche, 399-451.

Arcangeli F. 1970: Natura ed espressione nell'arte bolognese ed emiliana, Bologna.

Aronberg Lavin M. 1975: Seventeenth-Century Barberini Documents and Inventories of Art, New York.

Baglione, G. 1642: Le vite de' pittori scultori et architetti del pontificato di Gregorio XIII del 1572 in fino ai tempi di Papa Urbano VIII, nel 1642, Roma.

Barocchi P. 1960-1962: Trattati d'arte del Cinquecento, Bari, II.

Barroero L. 1983: "Antonio Pomarancio tra i due giubilei, 1600-1625”, Bollettino d'Arte, 1-16.

Barroero L. 1984: A proposito di Antonio Pomarancio, in M. Natale (ed.), Scritti di storia dell'arte in onore di Federico Zeri, Milano, II, 513-523.

Bell J., Willette T. (eds.) 2002: Art History in the Age of Bellori - Scholarship and Cultural Politics in Seventeenth Century Rome, Cambridge.

Benati D. 2006: 'L'Accademia degli Incamminati: una difficile affermazione", in D. Benati, E. Riccomini (a cura di), Annibale Carracci, Milano, 126-133.

Benati D., Bona Castellotti M. 1993: Guido Cagnacci, Milano.

Benati D., Paolucci A. (a cura di) 2008: Guido Cagnacci protagonista del Seicento tra Caravaggio e Reni, Cinisello Balsamo.

Bentini J. et al. (a cura di) 2006: Pinacoteca Nazionale di Bologna - Catalogo generale - 2 - Da Raffaello ai Carracci, Venezia.

Bernardini M.G. 2002: L'Oratorio del Gonfalone a Roma - Il ciclo cinquecentesco della Passione di Cristo, Cinisello Balsamo.

Bernini Pezzini G., Massari S., Prosperi Valenti Rodinò S. (eds.) 1985: Raphael Invenit, Roma.

Bianchi I. 2014: "Note sul collezionismo bolognese del Seicento attraverso documenti inediti: Bartolomeo Dolcini e i 'quadri di pittura fatti per mano di Ludovico Carrazzi"”, in G. Perini Folesani, A. Ambrosini Massari (a cura di), Riflessi del collezionismo tra bilanci critici e nuovi contributi / Reflections of/on art collecting, between critical assessments and new contributions, Firenze, 145-158.

Bodmer H. 1939: Ludovico Carracci, Burg bei Magdeburg, 1939 (trad. it. parziale, limitata al solo saggio introduttivo, pubblicata in «Accademia Clementina - Atti e Memorie», 1993, 43-116).

Bohn B. 2004: Ludovico Carracci and the Art of Drawing, New York.

Campbell Hutchinson J. (ed.) 1996: The Illustrated Bartsch - 8 - Early German Artists: Martin Schongauer, Ludwig Schongauer and copysts, New York.

Casciello A.M. 2018: "Per l'iconologia del Profeta Elia di Girolamo Savoldo", RIASA, 245-281.

Danesi Squarzina S. 2003: La collezione Giustiniani Gli inventari, I.

De Marchi A. 2016: Collezione Doria Pamphilj Catalogo generale dei dipinti, Cinisello Balsamo.

Dolcini B. 1631: De vario Bononiae statu, Bologna, Ferroni.

Emiliani A. 1984: "Le Storie di Giasone: invenzione e forma", in A. Emiliani (ed.), Bologna 1584. Gli esordi dei Carracci e gli affreschi di Palazzo Fava, Bologna, XVII-LXX.

Emiliani A. (a cura di) 1993: Ludovico Carracci, Bologna.

Emiliani A. 2011: Le Storie di Giasone in Palazzo Fava a Bologna - Ludovico, Agostino e Annibale Carracci, Bologna.

Faietti M., Hernad B. 1993: Incisori tedeschi e fiamminghi del secolo XV - La collezione della Pinacoteca Nazionale di Bologna, Bologna.

Fanti M. 1980: I macellai bolognesi: mestiere, politica $e$ vita civile nella storia di una categoria attraverso $i$ secoli, Bologna.

Fara G.M. 2007: Albrecht Dürer: originali, copie, derivazioni, Firenze.

Feigenbaum G. 1985: Ludovico Carracci: A Study of his Later Career and a Catalogue of his Paintings, Ann Arbour, UMI, (tesi di PhD discussa all'Università di Princeton nel 1984).

Feigenbaum G. 1993: Scheda n.19, in A. Emiliani (a cura di), Ludovico Carracci, Bologna, 42-43.

Fortunati V. 1994: Lavinia Fontana, 1552-1614, Milano.

Freeman Bauer L. 1983: "L'inventario dei beni di Antonio Pomarancio e alcune note sulla vita e l'opera del pittore", Bollettino d'Arte, 31-34. 
Gheza Fabbri L. 1988: L'organizzazione del lavoro in una economia urbana - Le Società d'Arti a Bologna nei secoli XVI e XVII, Bologna.

Giffi E. 1983: "Alcune proposte per Antonio Pomarancio", Bollettino d'arte, 17-30.

Guarino S., Masini P. 2006: Pinacoteca Capitolina Catalogo Generale, Milano.

Grasman E. 2009: "On closer inspection: the interrogation of Paolo Veronese", Artibus et historiae, 125134.

Haskell F., Penny N. 1982: Taste and the Antique, New Haven and London.

Hermann Fiore K. (a cura di) 2007: Dürer e l'Italia, Milano.

Keazor H. 2007: "Il vero modo" - Die Malereireform der Carracci, Berlino, 185-187.

Malvasia C.C. 1678: Felsina Pittrice - Vite de' pittori bolognesi, Bologna, D. Barbieri, 1678, I.

Mancini G., Salerno L., Marucchi A. 1956: Considerazioni sulla pittura, Roma. Accademia Nazionale dei Lincei, I, 216-220.

Marino G.B. 1979: La Galeria, Padova, I.

Montalbani O. 1641 = I. Antonius Bumaldus 1641: Minervalia Bononiensium Civium Anademata seu Bibliotheca Bononiensis, Bologna, Benacci.

Montanari T. 1997: "Il Cardinale Decio Azzolino e le collezioni d'arte di Cristina di Svezia”, Studi secenteschi, 186-264.

Mosini G.A. [= Massani] 1979: "Prefazione" in Le Arti di Bologna di Annibale Carracci, Roma.

Nicoletti M.F. 2017: 'L'atto pratico dell'edificare è il vero fine dell'architettura: le perizie di Giacomo della Porta e Domenico Fontana per le volte della basilica di San Petronio a Bologna", in Quaderni dell'Istituto di Storia dell'architettura, 59-78.

Novelli M.A. 2008: Scarsellino, Milano.

Parma E. 1997: Perin del Vaga - l'anello mancante, Genova.

Perini G. 1990: Gli scritti dei Carracci, Bologna.

Perini G. 1993a: "L'effigie di Ludovico: contributo all'iconografia del Carracci maggiore", Accademia Clementina - Atti e memorie, 355-385.

Perini G. 1993b: “'L'uom più grande in pittura che abbia avuto Bologna: l'alterna fortuna critica e figurativa di Ludovico Carracci”, in A. Emiliani (a cura di), Ludovico Carracci, Bologna, 269-344.

Perini G. 1994: "Raccolta di testi inediti o rari su Ludovico Carracci”, in Accademia Clementina - Atti e memorie, 85-104.

Perini G. 2002: "Caravaggio e i Carracci: appunti su un intreccio di percorsi", in M. Calvesi, C. Volpi (a cura di), Caravaggio nel IV Centenario della Cappella Contarelli, Roma, 303-311.
Perini G. 2006: "Il fregio con le Storie di Giasone dipinto dai Carracci a Palazzo Fava, Bologna”, in M. Kokole, B. Murovec, M. Sasel Kos, M. Talbot (eds.), Mediterranean Myths from Classical Antiquity to the Eighteenth Century/Mediteranski miti od antike do 18. stoletja, Lubiana, 189-211.

Perini G. 2007: Arte in Europa, 1550-1650 - L'età dei conflitti religiosi, Torino.

Perini G. 2010-2011: “Qualche Memoriale anonimo di Carlo Cesare Malvasia: intrecci inosservati tra interessi storico-artistici e professione legale", Arte a Bologna - Bollettino dei Musei Civici d'Arte Antica, 312-327.

Perini Folesani G. 2010: "Un parere inedito di Bernardo Buontalenti sulla basilica di San Petronio a Bologna e una prospettiva riformata sugli inizi dei Carracci", Confronto, 168-186.

Perini Folesani G. 2013a: "Le Annunciazioni di Ludovico in prospettiva - Riflessioni in margine al ricercare del Carracci maggiore", Studiolo», 110-126.

Perini Folesani G. 2013b: "Riflessioni baroccesche tra Bologna e Urbino", in B. Cleri (a cura di), Barocci in bottega, Urbino, 3-43.

Perini Folesani G. 2014a: "Termini di dissenso: spie lessicali di differenze critiche ed estetiche tra Malvasia e Bellori", in E. Oy-Marra, M. von Bernstoff, H. Keazor (Hrsg.), Begrifflichkeit, Konzepte, Definitionen - Schreiben über Kunst und ihre Medien in Giovan Pietro Belloris Viten und der Kunstliteratur der Frühen Neuzeit, Wiesbaden, 127-138.

Perini Folesani G. 2014b: "La vita dei Carracci di Carlo Cesare Malvasia: appunti in margine", in M. Visioli (a cura di), La biografia d'artista tra arte e letteratura - Seminari di letteratura artistica, Pavia, 77-100.

Perini Folesani G. 2015: "La triade carraccesca in Sandrart: un'interpretazione originale", in S. Meurer, A. Schreurs-Moret, L. Simonato (Hrsg.), Die Künstler der "Teutschen Academie" von Joachim von Sandrart: Aus Aller Herren Länder, Turnhout.

Perini Folesani G. 2016: "Effetti collaterali del Nicodemismo: i Carracci in bilico tra eterodossia ed ortodossia, tra arte e scienza", in D. Frascarelli (a cura di), L'altro Seicento. Arte a Roma tra eterodossia, libertinismo e scienza, Roma, 197-206.

Perini Folesani G. 2020a: "Borderline Iconographies: The Limits of Visual Communication in Conveying Christian Dogmas in Early Modern Europe", in S. Maltseva, A. Zacharova, E. Staniuchovic-Denisova (eds.), Actual Problems of Theory and History of Art - VIII - Collection of articles, 32-48.

Perini Folesani G. 2020b: "Conoscere è ricordare, ovvero: se gli asini volano", Teca 2020, 155-164. DOI: https://doi.org/10.6092/issn.2240-3604/11688. 
Perini Folesani G. 2020c: Sir Joshua Reynolds in Italia - Il soggiorno romano - I - Il taccuino di Plymouth, Firenze.

Perini Folesani G. 2021: "L'apparato illustrativo della Felsina Pittrice: riflessioni su un prodotto editoriale della storiografia secentesca", in Kritiké 2021 (in corso di stampa).

Pierguidi S. 2014: "Le aporie della critica di Malvasia tra difesa del primato lombardo e teoria eclettica", ArtItalies - La revue de l'AHAI, 68-78.

Puglisi C. 1999: Francesco Albani, New Haven and London.

Salerno L. 1988: I dipinti del Guercino, Roma.

Sandrart J. von 1675-1680: Teutsche Academie der Bau-, Bild- und Mahlerey Künste, NorimbergaFrancoforte, J. von Sandrart, M. Merian, 1675-1680, II.
Sandrart J. von 1683: Academia Nobilissimae Artis Pictoriae, Norimberga e Francoforte, C.S. Froberg, Eredi di J.F. Endterorum.

Stanzani A. 1993: "Regesto della vita e delle opere", in A. Emiliani (a cura di), Ludovico Carracci, Bologna, 199-268.

Zapperi R. 1986: "The Summons of the Carracci to Rome: Some New Documentary Evidence", The Burlington Magazine, 790-795.

Zapperi R. 1989: Annibale Carracci - Ritratto di artista da giovane, Torino.

Zeri F. 1959: Galleria Pallavicini in Roma - Catalogo dei dipinti, Firenze. 


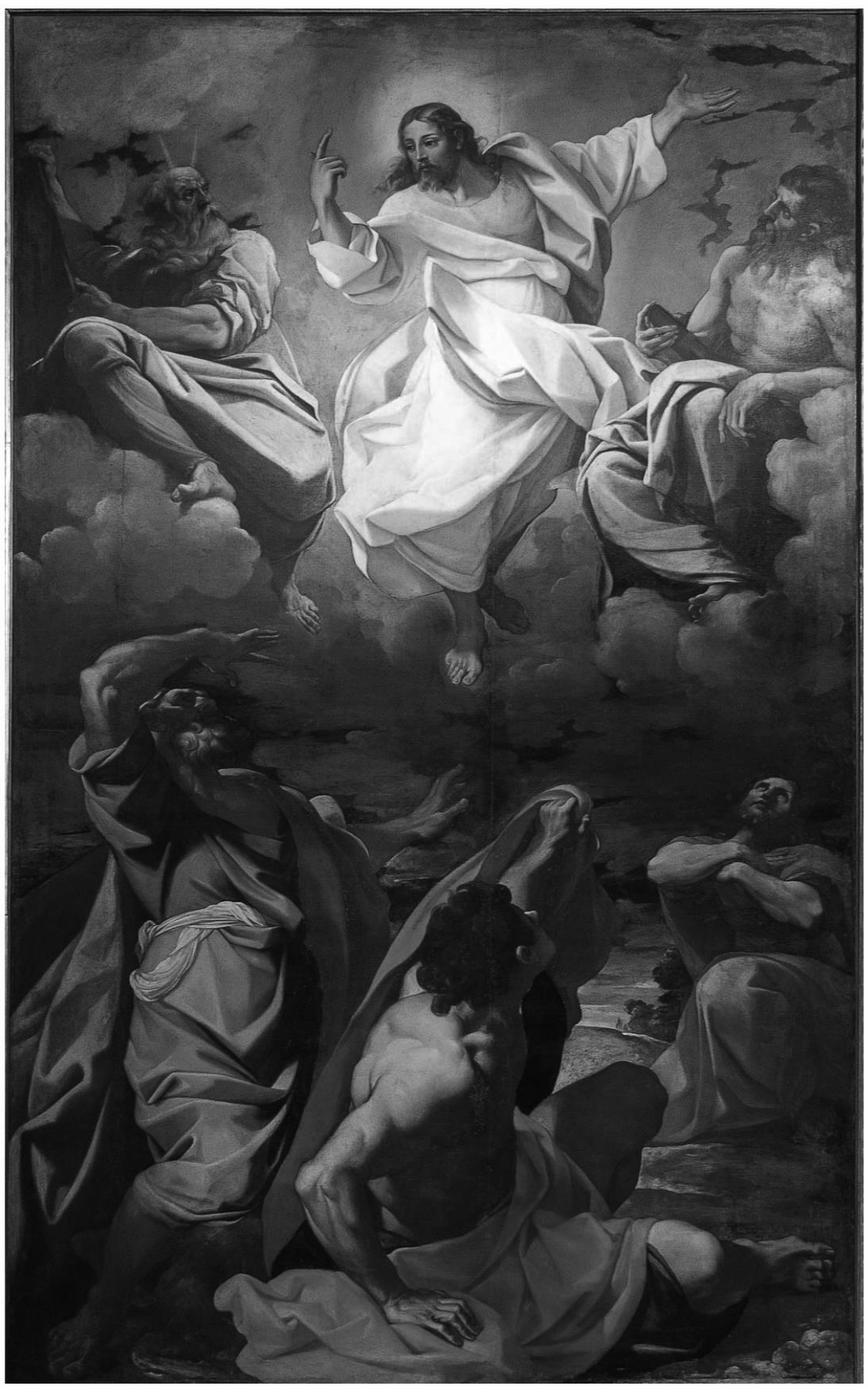

FIG. 1 - Ludovico Carracci, Trasfigurazione (Bologna, Pinacoteca Nazionale), 1595-1596. 


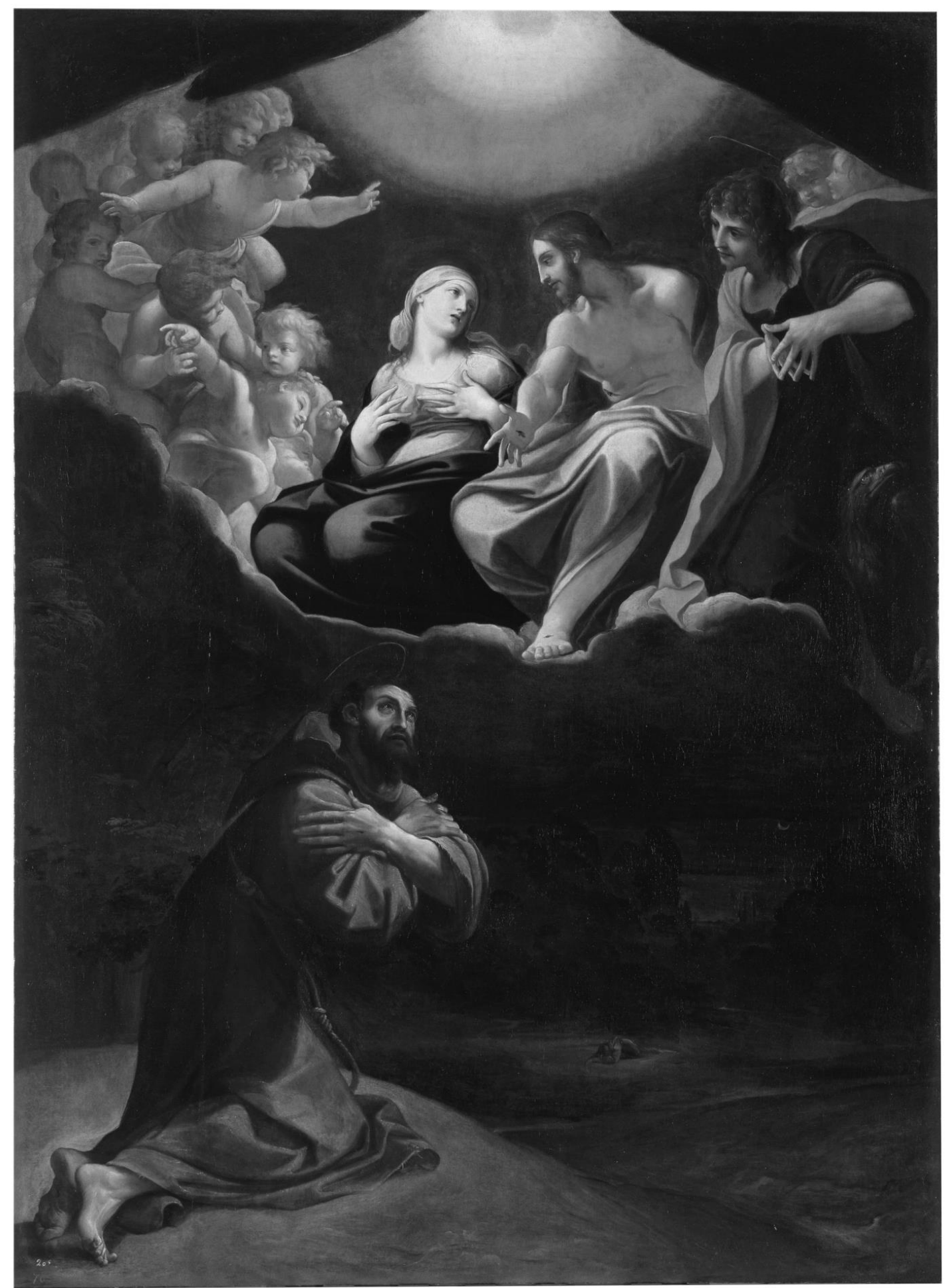

FIG. 2 - Ludovico Carracci, Visione della Porziuncola (Madrid, Museo del Prado), 1601-1603. 




FIG. 3 - Ludovico Carracci, Madonna con Bambino e Santi (Madonna Giustiniani) (New York, Metropolitan Museum of Art), 1607. 


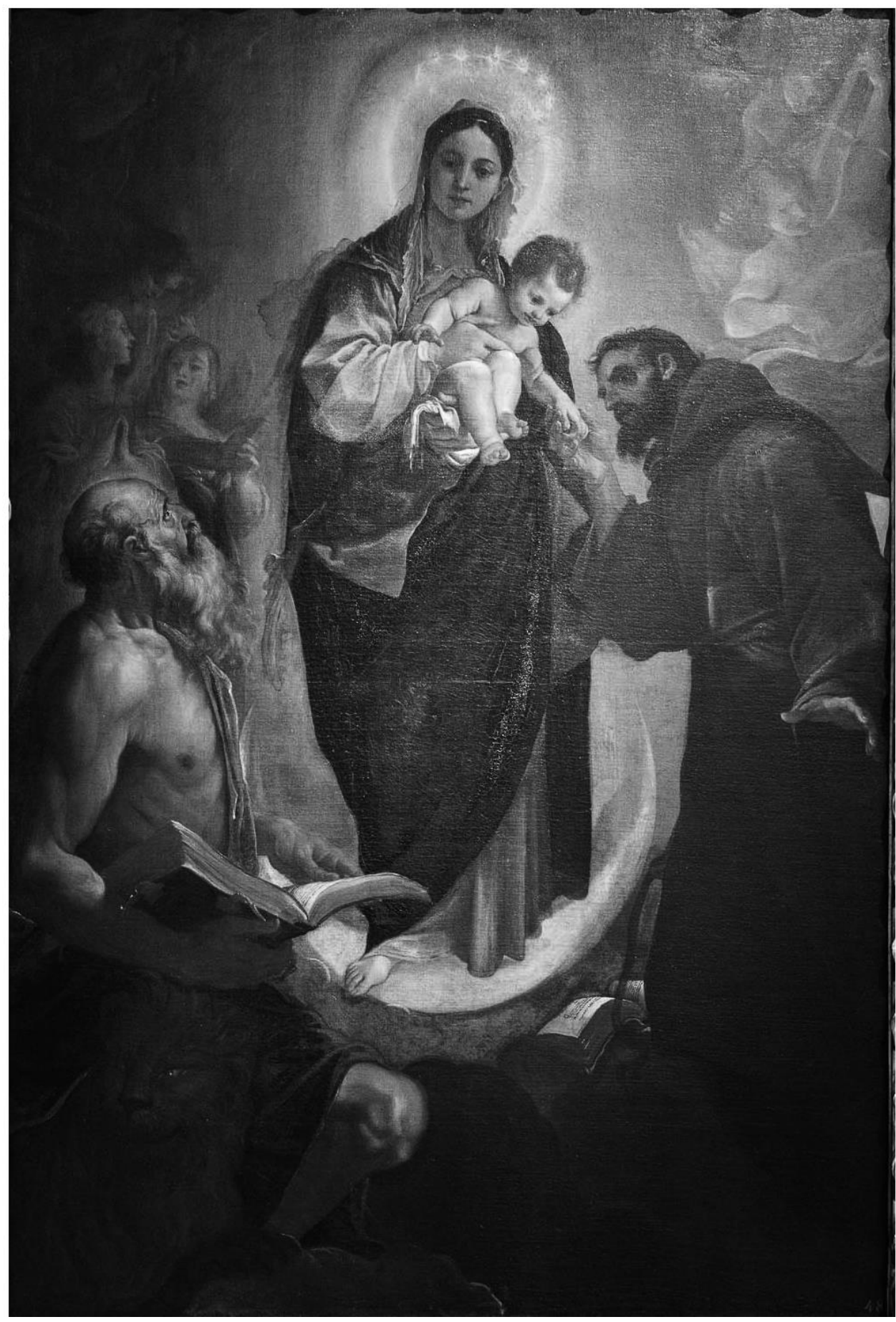

FIG. 4 - Ludovico Carracci, Madonna con Bambino e i santi Girolamo e Francesco (Madonna degli Scalzi) (Bologna, Pinacoteca Nazionale), circa 1590. 


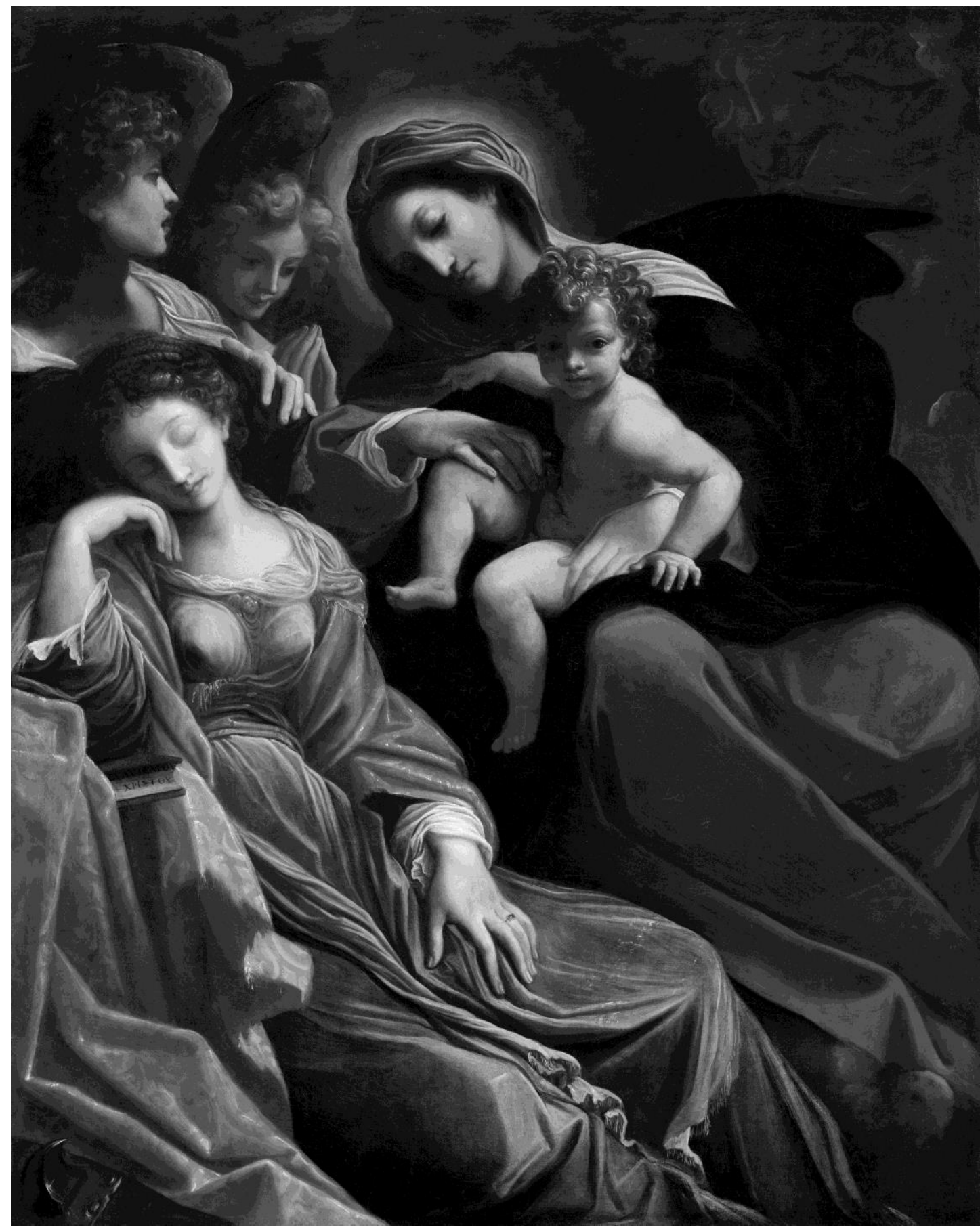

FIG. 5 - Ludovico Carracci, Sogno di Santa Caterina di Alessandria (Washington, National Gallery of Art), circa 1593. 
